\title{
Wissenschaftstheorie und Wissenschaftsgeschichte
}

\section{Kommentar zu Reinhard Mocek: Von der Universalität der Wissenschaftsgeschichte (1981)}

Jürgen Mittelstraß

Wissenschaft ist erstens eine besondere Form der Wissensbildung, eben die besonderen, strengen Normen und Standards folgende wissenschaftliche Wissensbildung. Sie ist zweitens eine Institution, nämlich eine gesellschaftliche Veranstaltung zur Stabilisierung der wissenschaftlichen Form der Wissensbildung. Und Wissenschaft ist drittens eine Idee und eine Lebensform, die auf (der Suche nach der) Wahrheit beruht und die allein die immer wieder eingeklagte Autonomie der Wissenschaft in ihren Institutionen begründet. Damit ist Wissenschaft auch in ihrem Werden stets eine Entwicklung in epistemischer, institutioneller und praktischer Hinsicht beziehungsweise hat jede historische Betrachtung diese unterschiedlichen Aspekte oder Bedeutungen zu berücksichtigen. Andernfalls bliebe das eine vom anderen isoliert, könnte es so erscheinen, als handele es sich in einer wissenschaftlichen Entwicklung allein um einen Erkenntnisfortschritt oder allein um einen institutionellen Vorgang, etwa unter politischen oder wirtschaftlichen Maßstäben. Der dritte Aspekt, Wissenschaft als Idee und Lebensform, bliebe in der Regel im Blick auf die moderne Wissenschaftsentwicklung

Der Nachdruck des Originalbeitrags ist in diesem Heft dem Kommentar vorangestellt und online unter dem DOI: 10.1007/s00048-010-0034-1 zu finden. 
ohnehin unberücksichtigt oder würde allenfalls als Teil des ersten Aspekts, als Teil einer besonderen Form der Wissensbildung, betrachtet.

Reinhard Mocek ist ein Autor, der alle drei Aspekte miteinander zu verbinden weiß. Das mag daran liegen, dass er Wissenschaftshistoriker und Philosoph in einer Person ist, dem das Historische nicht zur isolierten Kategorie gerät. Vielmehr tritt dieses stets philosophisch - im weiteren wie im engeren, wissenschaftstheoretischen Sinne - reflektiert auf, wie auch umgekehrt in seinem Denken alles Philosophische und Wissenschaftstheoretische Gegenstand auch einer historischen Reflexion ist. So in seinem 1981 veröffentlichten Vortrag „Von der Universalität der Wissenschaftsgeschichte", in dem in programmatischer Form der Status der Wissenschaftsgeschichte zur Diskussion steht, zugleich ein Konzept, das Mocek wenige Jahre später in seiner schönen Monographie Neugier und Nutzen weiter ausgearbeitet hat (Mocek 1988). Im Besonderen geht es - nicht ungewöhnlich in einer von der Dominanz marxistischer Fragestellungen geprägten philosophischen Umgebung - um das Konzept beziehungsweise das Programm einer Wissenschaftsgeschichte auf dem Hintergrund des Lenin'schen Postulats, in der Geschichte einen gesetzmäßigen Gang nachzuweisen.

Mocek verweist mit Blick auf die Wissenschaftsgeschichte auf die mageren Früchte dieses Programms (S. 112), will sich aber nicht gleich von ihm verabschieden. Nach seiner Vorstellung sind die Möglichkeiten weiterer ,hypothetischer Ansätze' nicht erschöpft, und auch in der damaligen Diskussion um das Kuhn'sche Paradigmenkonzept beziehungsweise die Leistungsfähigkeit einer rationalen Rekonstruktion der Wissenschaftsgeschichte sieht er, bestens vertraut mit der internationalen Theoriediskussion, die Chance, mit der Annahme gesetzmäßiger Strukturen der Wissenschaftsgeschichte weiterzukommen. Es geht ihm um eine Theorie der Wissenschaftsgeschichte, die sich nicht in der Beschreibung der Tätigkeit des Wissenschaftshistorikers erschöpft, und die gleichzeitig geeignet ist, der Wissenschaftstheorie zu historischer Plausibilität beziehungsweise zur historischen Bestätigung ihrer Geltungsansprüche zu verhelfen. Allerdings steht Mocek mit seinen Vorstellungen theoretisch in bestimmter Weise auch im Gegensatz zu den Konzepten um Kuhn, insofern bei diesem das wissenschaftstheoretische Interesse an der Wissenschaftsgeschichte gerade darin besteht, Vorstellungen einer Fundierung der Wissenschaft in einer theoretischen Begründungspraxis den Wind aus den Segeln zu nehmen. Die Beantwortung von Geltungsfragen soll nach Kuhn im Wesentlichen auf der Basis faktischer wissenschaftlicher Entwick- 
lungen erfolgen. Wissenschaftshistorischen Analysen käme sowohl unter Gesichtspunkten einer Theoriegeschichte als auch unter Gesichtspunkten einer Geschichte der Forschungspraxis (ihre gesellschaftliche Organisation eingeschlossen) die Funktion $\mathrm{zu}$, diese Konzeption zu bestätigen (weshalb nach Kuhn auch Wissenschaftstheorie und Wissenschaftssoziologie theoretisch und faktisch ineinandergehen).

Mocek wiederum sieht gerade in der möglichen Bestätigung begründungstheoretischer Ansprüche durch wissenschaftshistorische Analysen die Bedeutung einer systematischen Zusammenführung von Wissenschaftstheorie und Wissenschaftsgeschichte. Die Rede von ,Gesetzesforschung' ist dafür nur ein anderer Ausdruck, wenn auch problematischerweise (und sicher gegen seine eigenen Intentionen) an den älteren Positivismus Auguste Comtes und an den Logischen Empirismus, der selbst ein Begründungsprogramm verfolgte, erinnernd. Insofern steht er mit seinen Vorstellungen eher auf Seiten von Lakatos, den er in einem Atemzug mit Kuhn erwähnt, und, sofern es um Fragen eines Rekonstruktionsansatzes geht, auf Seiten des Erlanger Konstruktivismus (Lorenzen u. a.) in der Wissenschaftstheorie (vgl. Mocek 1988: 70-108). Nur geht es ihm nicht um eine rekonstruktive Geltung, das heißt um Geltungsansprüche, die sich auf die rekonstruktiven Leistungen einer wissenschaftstheoretischen Analyse beziehen, sondern um theoretische Wahrheit: ausdrücklich mahnt er gegenüber Jürgen Kuczynski, Siegfried Wollgast und anderen die Realisierung beziehungsweise Bestätigung der „Idee einer Gesetzmäßigkeit der Wissenschaftsentwicklung” (S. 113) an. Gleichzeitig sieht er auch in Kuczynskis Arbeitsprogramm (1974), an die Adresse des Wissenschaftshistorikers gerichtet - darunter die Analyse gesellschaftlicher Praxen als Ursachen der Wissenschaftsdynamik und die methodologische Analyse von Erkenntnisfortschritten -, mehr aber noch in Wollgasts Plädoyer (1976) für eine Verbindung von Wissenschafts- und Philosophiegeschichte, Ansätze zu einer an gesetzlichen Beziehungen orientierten Wissenschaftsforschung.

Die Frage ist nur, wie man zu derartigen Konzeptionen kommt, die nicht nur hypothetisch (oder rekonstruktiv), sondern in Analogie zu den Naturgesetzen gelten. Die Antwort sucht Mocek im Begriff der Universalität, das heißt in einem universellen Anspruch der Wissenschaftsgeschichte: „Geschichte der Wissenschaft ist nicht nur ein sich gesetzmäßig entwickelndes Ganzes, sondern gleichzeitig organischer Bestandteil der materiellen und geistigen Kultur der jeweiligen Epoche, ein Teil der allgemeinen Wissenschaftsgeschichte" (S. 113). Einmal abgesehen davon, dass auch hier 
wieder der Begriff eines sich ,gesetzmäßig entwickelnden Ganzen” schon als gegeben beziehungsweise als in sich vernünftig unterstellt wird, öffnen sich in einer derartigen Bestimmung und Dimensionierung die Aufgaben einer Wissenschaftsgeschichtsschreibung hin zu einer Universalgeschichte, die - auch wenn hier zu Recht auf Interdependenzen aufmerksam gemacht wird, die in der Wissenschaftsgeschichtsschreibung berücksichtigt werden müssen - weder vom Wissenschaftshistoriker noch vom Wissenschaftstheoretiker und auch nicht der Vereinigung beider geleistet werden können. Hier lugt das marxistische Programm aus allen systematischen Ritzen, getreu des auch von Mocek zitierten Diktums Lenins, dass die „Fortführung des Werks von Hegel und Marx [...] in der dialektischen Bearbeitung der Geschichte des menschlichen Denkens, der Wissenschaft und der Technik bestehen" muss (Lenin 1955-1971: 137, Hervorhebung, J. M.)

Mocek selbst scheint diesem Diktum beziehungsweise seinen Realisierungschancen nicht zu trauen. Das macht die für die schwierige politische und intellektuelle Situation, in der Mocek arbeitete, erstaunliche Bezugnahme auf Humanismus und Deutschen Idealismus, ferner auf den Legitimationsanspruch der Philosophie in diesem Kontext deutlich. Diesen Anspruch sieht auch er zwar erst durch den dialektischen und historischen Materialismus - der These, nicht der Realität nach! (S. 115) - eingelöst, doch ist unübersehbar, dass Mocek hier im Sinne der zu Beginn getroffenen Unterscheidung zwischen unterschiedlichen Bedeutungen von Wissenschaft deren Bedeutung als Idee im Auge hat und dabei Theorie ganz im Aristotelischen Sinne als höchste Form der Praxis ansieht. Das lässt sich zwar immer noch gut marxistisch deuten - und warum, wenn man Marx mit offenen Augen, das heißt ohne ideologische Scheuklappen, liest, auch nicht? -, weist aber im Grunde, etwa mit der für seine Umgebung provokatorischen Vermutung eines ,,einschlägigen Theoriedefizits der marxistischen Wissenschaftsgeschichte" (S. 116), über eine solche Deutung schon wieder hinaus.

Was Mocek selbst - in einer subtilen Kritik an einer Theorie, die nicht dort „lebt”, wo es um die Realität (hier die Realität der Wissenschaft und ihrer Geschichte) geht (ebd.) - als Theorie der Wissenschaftsgeschichte skizzenhaft ins Auge fasst, zielt denn auch in erster Linie auf eine Theoriediskussion, die wohl in einem marxistischen Rahmen (hier spricht er von „methodologischen Fundamenten"), aber mit offenem Resultat geführt werden soll. Genauer gesagt ist es auch hier die Kuhn-Diskussion hinsichtlich des in ihr dokumentierten Zusammengehens von wissenschaftstheoretischen und wissenschaftshistorischen Aspekten, die für 
Mocek, konkret bezogen auf die Stellung der Wissenschaftsgeschichte in einem disziplinären Kontext, nach einem neuen theoretischen Ansatz ruft. Und auch seine Analyse, warum die mit Humanismus und Deutschem Idealismus beschworene Idee der Wissenschaft und ihrer Geschichte nicht durchgehalten werden konnte, ist korrekt:

Der geistige Kosmos brach auseinander, weil sich die moderne Naturwissenschaft mit der Einbindung in einen als abgeschlossen geltenden philosophischen Horizont nicht abfinden konnte. Das Buch der Natur war für diese Wissenschaft ausschlaggebend, nicht das Zu-sich-selber-Kommen des objektiven Geistes. (S. 114)

Oder anders formuliert: dieser Kosmos brach auseinander, „,weil der philosophische Idealismus auf die Dauer die praktisch orientierte Naturwissenschaft nicht in sich bewahren konnte" (ebd.). Ob das mit einer marxistischen Philosophie besser zu bewerkstelligen wäre? Es wäre aufschlussreich zu sehen, wie Mocek heute, wo die Theoriediskussion um Wissenschaft und ihre Geschichte wieder als eine völlig offene geführt wird, darüber denkt.

Worum es Mocek in seiner Stellungnahme zur Universalität der Wissenschaftsgeschichte jenseits aller philosophischen Voraussetzungen und Festlegungen geht, sind drei Dinge: (1) Die disziplinäre Selbständigkeit der Wissenschaftsgeschichte, (2) die Einheit von Philosophie- und Wissenschaftsgeschichte, ausgedrückt in der Einheit von Wissenschaftstheorie und Wissenschaftsgeschichte, (3) die Notwendigkeit einer Theorie der Wissenschaftsgeschichte. Dies sind in der Tat auch heute noch wesentliche Themen zwischen Philosophie und Wissenschaft. Vor allem die angemahnte Theoriediskussion ist heute so wichtig wie 1981, als Mocek seinen Vortrag über die Universalität der Wissenschaftsgeschichte hielt.

\section{Literatur}

Kuczynski, Jürgen, 1974, Prolegomena zu einer Geschichte der Wissenschaft. Berlin (Ost): Akademie-Verlag [=Sitzungsberichte der Akademie der Wissenschaften der DDR, 5].

Lenin, Wladimir I., 1955-1971. Konspekt zu Hegels „Wissenschaft der Logik” Werke, I-XL, hg. vom Institut für Marxismus-Leninismus beim Zentralkomitee der SED, Berlin (Ost), XXXVIII, 77-229.

Mocek, Reinhard, 1988, Neugier und Nutzen. Blicke in die Wissenschaftsgeschichte. Berlin (Ost): Dietz Verlag.

Wollgast, Siegfried, 1976, Zum Verhältnis von Wissenschafts- und Philosophiegeschichte, Wissenschaftliche Zeitschrift der Humboldt-Universität, 1 [=Gesellschafts- und Sprachwissenschaftliche Reihe, 25], 37-42. 
Jürgen Mittelstraß

Konstanzer Wissenschaftsforum

Universität Konstanz

78457 Konstanz

Deutschland

E-Mail: Juergen.Mittelstrass@uni-konstanz.de 